\section{Neurofluids: A holistic approach to their physiology, interactive dynamics and clinical implications for neurological diseases}

\author{
Nivedita Agarwal, ${ }^{1,2}$ \\ Christian Contarino, ${ }^{3}$ Eleuterio F. Toro ${ }^{4}$ \\ ${ }^{1}$ Section of Radiology, Hospital Santa \\ Maria del Carmine, Rovereto (TN), \\ Italy; ${ }^{2}$ Center for Mind/Brain Sciences \\ CIMeC, University of Trento, Italy; \\ ${ }^{3}$ Computational Life Inc., Delaware, \\ USA; ${ }^{4}$ Laboratory of Applied \\ Mathematics, University of Trento, Italy
}

\begin{abstract}
There is increasing interest in understanding the physiology of the extracellular fluid compartments in the central nervous system and their dynamic interaction. Such interest has been in part prompted by a vigorous resurgence of the role of the venous system, the recent discoveries of the meningeal lymphatics, the brain waste removal mechanisms and their potential link to neurological diseases, such as idiopathic intracranial hypertension, Ménière's disease, migraine, small vessel disease, and most neurodegenerative diseases.
\end{abstract}

The rigid cranial cavity houses several space-competing material compartments: the brain parenchyma (BP) and four extracellular fluids, namely arterial, venous, cerebrospinal fluid (CSF) and interstitial fluid (ISF). During cardiac pulsations, the harmonious, temporal and spatial dynamic interaction of all these fluid compartments and the BP assures a constant intracranial volume at all times, consistent with the Monro-Kellie hypothesis. The dynamic interaction involves high-pressure input of arterial blood during systole and efflux of CSF into the spinal subarachnoid space (SSAS) followed by venous blood exiting directly into the vertebral and internal jugular veins towards the heart and intraventricular CSF displacing caudally towards the SSAS. Arterial pulsatile energy is transmitted to the BP that contributes to the smooth movement of fluids in and out of the brain. Perturbing any of these fluid compartments will alter the entire brain dynamics, potentially increase intracranial pressure, affect perfusion and hamper clearance capacity of metabolic waste.

This review of all major extracellular fluid compartments within the brain, advocates a holistic approach to our understand- ing of the fluid dynamics, rather than focusing on a single compartment when analyzing neurological diseases. This approach may contribute to advance our comprehension of some common neurological disorders, paving the way to newer treatment options.

\section{Introduction}

Neurological disorders constitute a major public health challenge and are associated with life-long disability. ${ }^{1}$ Much progress has been made in understanding the etiopathogenesis of many of these, however, there are still several gaps in our knowledge of many others, some of which are still considered idiopathic (a disease of unknown etiology).

The Monro-Kellie hypothesis formulated by the Scottish anatomist Alexander Monro (secundus) and his former student George Kellie in 1783 remains a cardinal principle in understanding the physiology of the central nervous system (CNS). ${ }^{2}$ This hypothesis, as originally stated, maintains that: i) brain contents are enclosed in a nonexpandable bony skull; ii) the volume of skull contents is constant at all times; iii) the brain parenchyma (BP) is largely incompressible; and iv) venous outflow must match the arterial blood inflow within the cardiac cycle. ${ }^{3}$ However, over the years, parts of the Monro-Kellie doctrine have been challenged largely because it ignored the presence of cerebrospinal fluid (CSF). We now know that in addition to venous outflow, caudal displacement of CSF contributes to counterbalance arterial blood input within the rigid bony skull during each cardiac cycle and these movements occur in a precise temporal order. ${ }^{4}$ These newer concepts help us better understand the dynamic interconnection between the various compartments and how anomalies in one can affect the dynamics of others. ${ }^{5}$ The cranium houses several space-competing material compartments: BP and four extracellular fluids, namely arterial, venous, interstitial fluid (ISF) and CSF. In light of recent renewed interest in fluids such as ISF, the discovery of the meningeal lymphatics ${ }^{6}$ and proposed mechanisms for brain waste clearance mechanisms, ${ }^{7-9}$ it has become necessary not only to revisit the original Monro-Kellie doctrine but also to comprehend the intricate interplay of the different CNS compartments.

We will do this by reviewing the relevant anatomy and physiology of the BP and single CNS extracellular fluid compartments (we will call them neurofluids). Key elements of such interactions and major possible neuro-
Correspondence: Nivedita Agarwal, Hospital S. Maria del Carmine, Corso Verona 4, 38068 Rovereto (TN), Italy.

Tel.: +39.0464.404066

E-mail: niveditaaga@gmail.com

Key words: Monro-Kellie; phase-contrast magnetic resonance imaging; neurofluids; chronic cerebrospinal venous insufficiency; glymphatic system; meningeal lymphatics: IPAD.

Contributions: NA, designed the paper to link mechanical aspects of neurofluid interaction and neurological diseases as well as wrote the manuscript; $\mathrm{CC}$, contributed to the revision of the paper and designed figures; EFT, contributed to the design of fluid mechanics framework of paper and holistic view of neurofluids as well as the writing of parts of the paper.

Conflict of interest: the authors declare no conflict of interest.

Received for publication: 7 August 20019.

Revision received: 31 August 2019.

Accepted for publication: 31 August 2019.

This work is licensed under a Creative Commons Attribution 4.0 License (by-nc 4.0)

C Copyright: the Author(s), 2019

Licensee PAGEPress, Italy

Veins and Lymphatics 2019; 8:8470

doi:10.4081/vl.2019.8470

logical diseases that may derive from specific alterations have been schematically illustrated in Figure 1. This will form the basis of our understanding of the proposed brain waste clearance mechanisms and how a holistic approach could help provide answers to still unsolved questions.

\section{Cerebral arterial system}

Paired internal carotid and vertebral arteries assure a steady blood supply to the cerebral hemispheres that anastomose at the level of the circle of Willis (Figure 2A). The cardiac input to the brain is approximately $15-20 \%$ of the total cardiac output. For a healthy adult weighing $70 \mathrm{kgs}$, the cardiac output is estimated at approximately 5 $\mathrm{L} / \mathrm{min}$ and brain arterial input is about 750 $\mathrm{mL} / \mathrm{min}$ to $1000 \mathrm{~mL} / \mathrm{min}$. Each of these arteries will divide progressively into smaller branches (pial or leptomeningeal arteries) as they reach the cortical surface in the cerebral subarachnoid space (CSAS). ${ }^{10}$

The cortical surface is lined by glia limitans, a structure that is made up of astrocyt- 


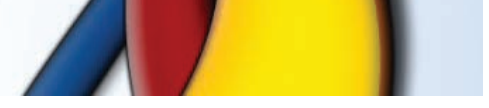

ic end-feet and tight junctions. The pia mater overlies the glia limitans and fuses with the basement membrane (BM) of the pial arteries forming the outer wall of a funnel-shaped CSF-filled space. ${ }^{11}$ Cortical pia mater is also reflected over these leptomeningeal arteries. ${ }^{12,13}$ The adventitial layer of leptomeningeal arteries is surrounded by perivascular spaces (PVS), or compartments which are fluid-filled but also contain connective tissue and cells (Figure 1). ${ }^{12,14}$ PVS are an important exchange route for CSF and ISF for substances including CSF-infused tracers to reach brain parenchyma alongside vessels. ${ }^{15}$

The arteriolar walls are lined with layers of smooth muscle cells (SMCs) that form the tunica media and have contractile properties that ensure a constant cerebrovascular tone. ${ }^{16}$ These are innervated by the autonomous nervous system or extrinsic innervation. Sympathetic innervation will cause vasoconstriction in response to an increase in arterial pressure, and reduce blood flow, whereas parasympathetic innervation will cause vasodilation of arterioles in a situation of low arterial pressure and increase blood flow. ${ }^{17}$ Capillaries are innervated by nerves arising within the brain. These are subcortical nuclei in the brainstem such as nucleus coeruleus, raphe nuclei and other nuclei in the basal forebrain. ${ }^{18}$ This intrinsic innervation is more pronounced at the astrocyte-neuronal junction. ${ }^{17}$ The contractile activities of SMCs and pericytes in the arterioles and capillaries are major actors that modulate lumen diameter thereby determining the entity of cerebrovascular resistance (CVR) to flow and assures constant cerebral perfusion pressure (CPP) through a unique process known as cerebral autoregulation (CA). ${ }^{19,20}$

According to Poiseuille's law, flow $Q$ through a vascular segment of length $L$ is directly determined by the pressure drop $\Delta P$ and inversely to the resistance to flow $R$ :

$$
Q=\frac{\Delta P}{R}
$$

where $R$ is

$$
R=\frac{8 \eta \mathrm{L}}{\pi r^{4}}
$$

$\eta$ is the blood viscosity and $r$ is the vessel radius. Thus, $Q$ becomes:

$$
Q=\frac{\Delta P \pi r^{4}}{8 \eta L}
$$

Equation 3, shows how a change in vessel radius will determine a flow change exponentially to the power of four making it a powerful mechanism to instantly regulate $Q$, or cerebral blood flow $(\mathrm{CBF})$.

$\mathrm{CPP}$ is defined as:

$C P P=M A P-\max \{I C P, J V P\}$ (4) where ICP is intracranial pressure, JVP is jugular venous pressure and MAP is mean arterial pressure. The normal range of MAP is $70-100 \mathrm{mmHg}$. Brain capillaries are lined with a single layer of continuous non-fenestrated endothelial cells that are held together with tight junctions and constitute the blood-brain barrier (BBB). ${ }^{21}$ Several specif-

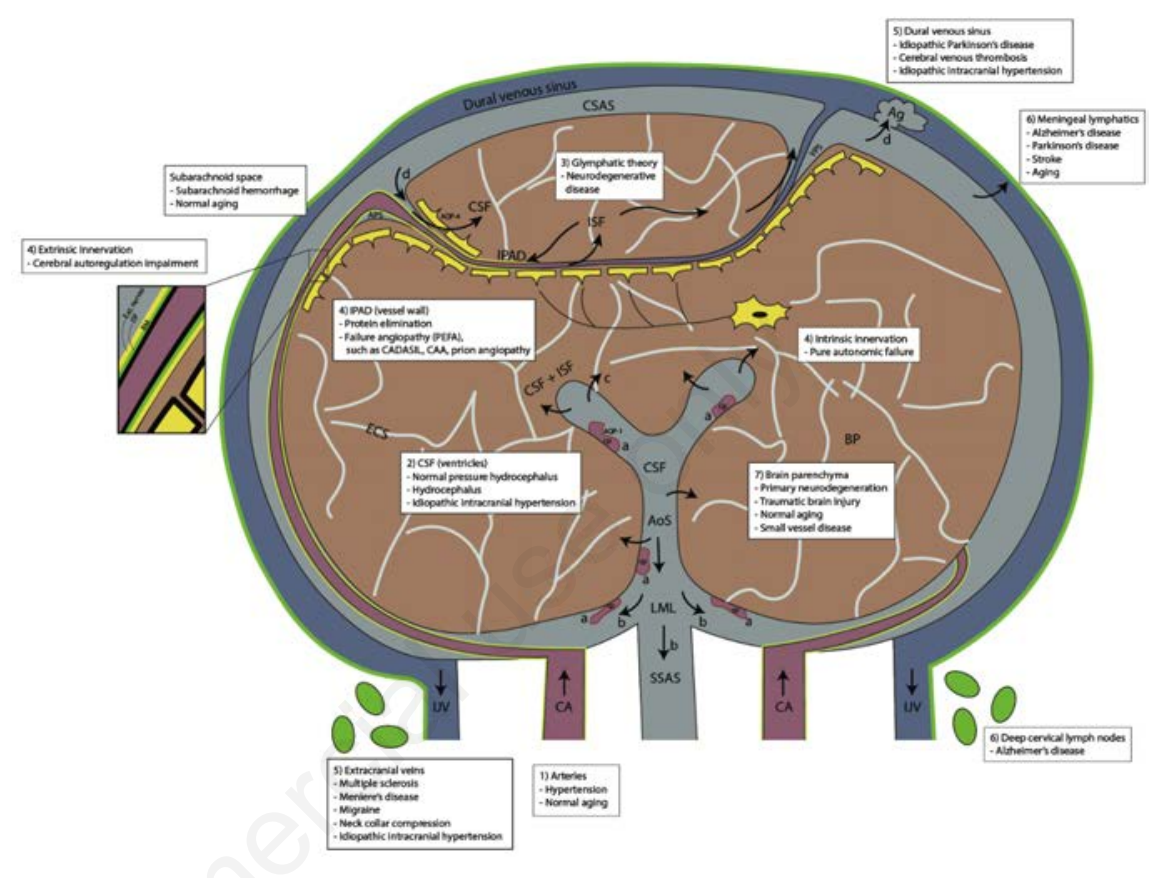

Figure 1. Schematic drawing on a coronal section of the brain illustrating the interaction between neurofluid compartments and brain parenchyma and possible neurological diseases due to their derangement: 1) Arterial system: branches of carotid arteries (CA) and vertebral arteries (not shown) reach the CSAS, the walls of which contain sympathetic innervation for smooth muscle cells (ext.nerves) 2) CSF pathway: a) CSF is produced via choroid plexi (CP) in the ventricles and flows caudally through the foramina of Luschka and Magendie towards the cerebral subarachnoid space(CSAS), b) CSF flows caudally and reaches the CSAS via foramen of Lushka and Magendie (LML) and towards the spinal (SSAS), c) CSF also moves through the ventricular ependymal wall towards the brain parenchyma (BP), d) CSF drains into the dural venous sinus through arachnoid granulations (Ag); e) CSF from the CSAS enters arterial perivascular space (APS) and into the BP through aquaporin 4 (AQP-4) water channels; $d$ ) CSF flows through the ventricular ependymal wall toward the brain parenchyma (BP) and extracellular space (ECS) where it intermingles with interstitial fluid (ISF); 3) Glymphatic theory: CSF from arterial perivascular space (APS) enters the BP via AQP-4 and mixes with ISF. Bulk flow and/or diffusion processes move ISF/CSF from the BP towards the venous perivascular space (VPS), transporting metabolic waste out towards the CSAS and into the dural venous sinus. 4) Intramural periarterial drainage (IPAD): ISF flows is in the opposite direction to arterial flow along the basement membranes (BM) of arteries (zoomed box) and leaves the intracranial cavity along the walls of the CA into the deep cervical lymph nodes. Extrinsic and intrinsic innervations lines the arterial and capillary wall respectively and determine cerebrovascular tone and contribute to cerebral autoregulation. 5) Venous system: bridging veins (BV) lie on the cortical surface in the CSAS and drain into the superior sagittal sinus (here shown as dural venous sinus). Venous blood flows out the intracranial cavity via internal jugular veins (IJV) and vertebral veins (not shown). 6) Meningeal lymphatics: lymphatic channels line dural venous sinus and provide an important pathway for CSF drainage towards the deep cervical lymph nodes. These can also be drained via perineural sheaths around cranial nerves and through the cribriform plate to reach deep cervical lymph nodes. 7) Brain parenchyma (BP) is made up of 80 $85 \%$ of cells and intracellular fluid, the rest is made up of extracellular space (ECS) and contains ISF. A potential list of diseases, already published in the literature, that might affect one or more compartments are listed in the various boxes. CADASIL $=$ cerebral autosomal dominant arterial with subcortical infarcts and leucoencephalopathy, $C A A=$ cerebral amyloid angiopathy, PEFA=protein elimination failure angiopathy. 
ic molecules form the unique tight junction, such as occludins, claudins and junctional adhesion molecules. ${ }^{22}$ BBB selectively restricts paracellular transport of solutes and cells across brain capillaries and limits dangerous fluctuations in local concentrations of ions, proteins, glucose, neuromodulators, and neurotransmitters and to prevent entry of pathogens and toxins inside the brain. $^{23}$

\section{Cerebrospinal fluid}

There are multiple controversies and lack of conclusive data in the literature regarding CSF dynamics. ${ }^{24}$ The historical understanding is that CSF is produced within the choroid plexi in the lateral, third and the fourth ventricles and flows through the foramen of Magendie and Luschka into CSAS and caudally towards the SSAS (Figure 1,2F). It is produced at a rate of 0.3$0.4 \mathrm{~mL} / \mathrm{min}^{25}$. Total CSF volume is approximately $189 \pm 29 \mathrm{~mL}$ in adults and varies with age and gender. ${ }^{26} \mathrm{CSF}$ subserves nutritional, immunologic, metabolic and clearance functions in addition to protecting the brain and spine from external injury. ${ }^{27}$

Choroidal capillaries are characterized by multiple fenestrations and are bounded by a single layer of choroidal epithelium. The CSF facing surface, or the apical surface of the epithelial cells, is lined with tight junctions and contain microvilli forming the blood-CSF barrier. ${ }^{28}$ Multiple ion channels and water ion channel aquaporin 1 (AQP-1) on the apical and the basolateral surfaces operate to create a favorable osmotic gradient through secretion of $\mathrm{Na}^{+}$, facilitating water movement through AQP-1 channels across the $\mathrm{CP}$ epithelium into the ventricles. ${ }^{29} \mathrm{CSF}$ is absorbed into the dural venous sinuses through subarachnoid granulations or villi that contain fine water channels in their core ${ }^{12}$ (Figure 2E). Thus, CSF also enters PVS and flows in a centripetal direction towards the BP via aquaporin 4 water channels (AQP-4) (glymphatic theory) $)^{7,30,31}$ (Figure 1). CSF also exits through the cribriform plate towards nasal lymphatics and the cranial cavity via major foramina of the skull base alongside cranial nerves, arteries, and veins towards the deep cervical lymph nodes ${ }^{8,31-33}$ (Figure 1). In addition, true meningeal lymphatics along the meninges surrounding the dural venous sinuses also drain CSF towards the deep cervical lymph nodes as observed in both animal $^{6,33}$ and human studies ${ }^{34}$ (Figure 1).

An alternative view of CSF dynamics, also known as the Bulat-Klarica-Oreskovic hypothesis, strongly advocates that CSF production and absorption occurs within the entire BP and that its formation in the choroid plexi and absorption in the dural venous sinuses may be of less importance. ${ }^{35}$ According to these authors, CSF is produced and absorbed at the capillary level through Starling forces acting between plasma and the extracellular fluid in the extracellular space (ECS). ${ }^{35,36}$ There is also evidence for transependymal CSF flow into the brain parenchymal ECS where it may intermingle with ISF. ${ }^{27,37,38}$

Intraventricular CSF flow is bidirectional, primarily driven by cardiac activity (expansion of choroidal arteries in the plexi), although it is also affected by breathing. ${ }^{39}$ During systole, CSF from the ventricular system flows caudally towards the SSAS (also known as, net negative flow) and flows cranially during diastole (also known as, net positive flow) with minimum net displacement in the cranial direction. CSF dynamics can be quantified using validated and more reliable cine phase-contrast flow magnetic resonance imaging (PCMRI) techniques..$^{40,41}$

\section{Cerebral venous system}

Three different venous drainage networks exist in the brain. An outer, superficial segment of veins that drain the scalp, muscle and tendons and an intermediate segment that serves the skull tables and the dura mater (diploic veins). ${ }^{42}$ The third set of veins are intra-parenchymal veins that may be further subdivided into superficial and deep veins (Figure 2B,C). Several anatomical variations are possible and veins can vary in number, size, symmetry and their drainage patterns. ${ }^{43}$ The different venous networks can communicate with each other according to physiological needs or pathological conditions (Figure 3C). Intracranial veins and sinuses are valveless allowing flow to reverse in the case of downstream venous obstruction. ${ }^{44}$ Bridging veins (BV) however, drain into dural sinuses at the level of arachnoid granulation through a cuff-like segment lined with circularly arranged $\mathrm{SMC}^{45}$ (Figure 2E). This segment remains closed until transmural pressure

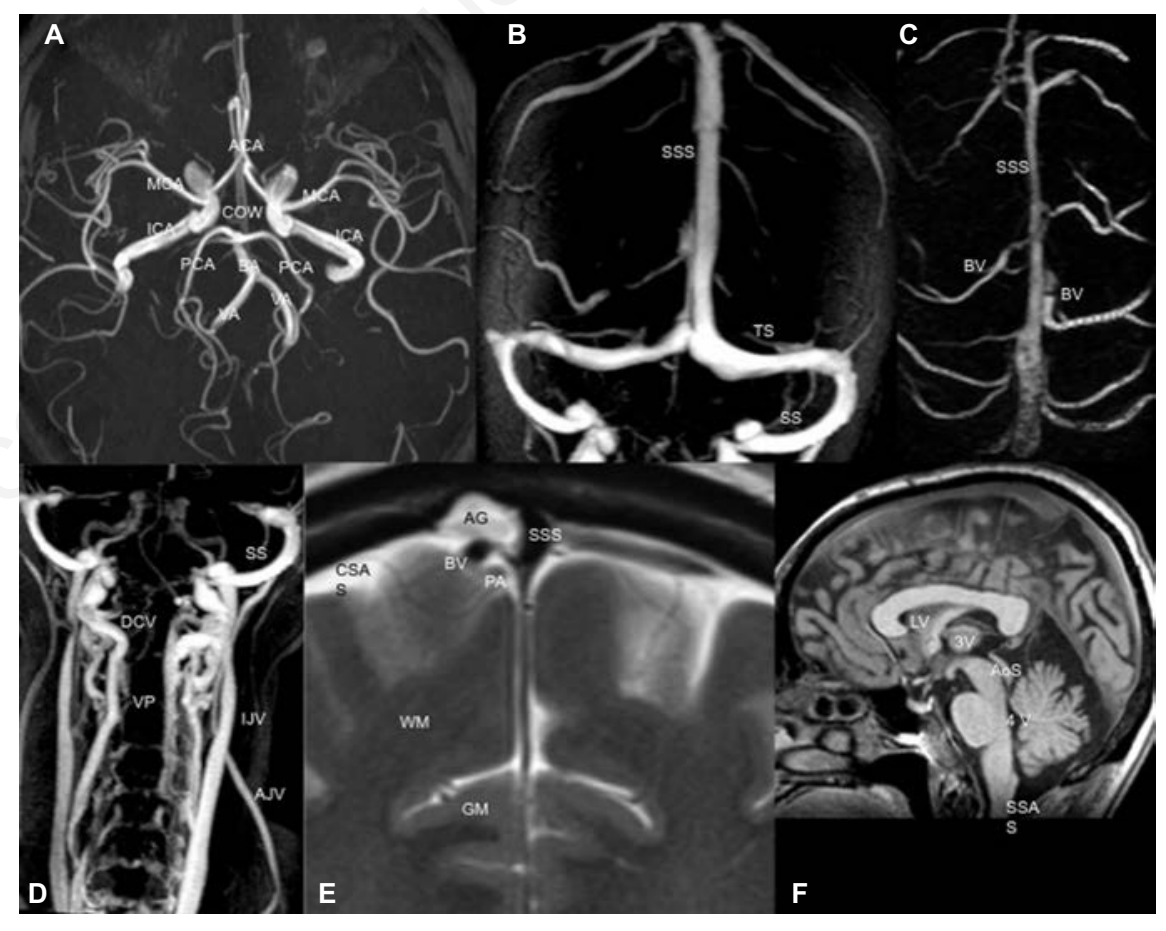

Figure 2. Normal anatomy. A) 3D time-of-flight (TOF) magnetic resonance angiography image illustrating the main intracranial arteries and the circle of Willis (COW). ICA=internal carotid artery, $\mathrm{MCA}=$ middle cerebral artery, $\mathrm{ACA}=$ anterior cerebral artery, $\mathbf{P C A}=$ posterior cerebral artery, $\mathrm{VA}=$ vertebral artery; $\mathrm{B})$ contrast enhanced magnetic resonance venography (CE-MRV) illustrating the main superficial dural venous sinuses. SSS=superior sagittal sinus, TS=transverse sinus, $S S=$ sigmoid sinus; $C$ ) $C E-M R V$ showing bridging veins (BV) or cortical veins; $\mathrm{D}) \mathrm{CE}-\mathrm{MRV}$ of the intracranial veins of the neck. $\mathrm{DCV}=$ deep cervical veins, $\mathrm{VP}=$ vertebral plexi, $\mathrm{IJV}=$ internal jugular vein, $\mathrm{AJV}=$ anterior jugular vein; E) T2 weighted coronal MRI illustrates the subarachnoid space and its principle contents. AG=arachnoid granulation, CSAS=cerebral subarachnoid space, WM=white matter, $G M=$ gray matter, $P A=$ pial artery; $F)$ T1 weighted sagittal image. $\mathrm{LV}=$ =lateral ventricle, $3 \mathrm{~V}=$ third ventricle, $A$ oS $=$ aqueduct of Sylvius, $4 \mathrm{~V}=$ fourth ventrice, SSAS=spinal subarachnoid space. 
builds up such that BVs expand and venous blood flows into the dural sinuses. ${ }^{46} \mathrm{BVs}$ are sensitive to transmural pressure. Increase in ICP can cause a collapse of such veins, whereas an increase in venous sinus pressure will cause engorgement of BV. In both cases venous outflow will be severely impacted (Figure 3).

Intracranial venous vessels are in direct continuity with extracranial neck vessels and the intra- and extra-spinal venous systems, also termed as the craniospinal venous system (CSVS) ${ }^{47}$ The CSVS is an extensive network of intricately communicating veins connected to the azygos, hemiazygos veins and both superior and the inferior vena cava ${ }^{47}$ Internal jugular veins (IJV) are considered the major recipients of intracranial venous blood from the sigmoid sinuses in around $72 \%$ of healthy individuals (jugular drainers) (Figure 2D) ${ }^{48}$

Veins carry oxygen-depleted blood towards the heart. They also represent the primary route for the removal of metabolic waste products from tissues. The role of venous vessels in maintaining ICP is underscored by the fact that these high capacitance vessels contain $70 \%$ of the entire intracranial blood volume such that both passive and active control of venous SMCs can cause important intracranial blood volume shifts, significantly affecting ICP. ${ }^{49}$ Veins are easily collapsible and the crosssectional area (CSA) of veins can be influenced by multiple internal and external factors. Internal factors include upstream and downstream pressure, flow rates and gravitational forces whereas the predominant external factor is the surrounding ICP. There is also an active neurogenic control of venous vessels via monoaminergic innervation..$^{50}$

Recalling Equation (1), venous flow:

$Q=\frac{P v-C V P}{R}$

where $P v$ is venous pressure, CVP is pressure within the right atrium (central venous pressure) and $R$ represents resistance to flow (largely determined by venous CSA). In the veins, $R$ is relatively low as they readily expand to allow venous blood to flow unhindered towards the heart. Both extracranial (e.g. IJV) and intracranial strictures (e.g. dural venous sinus) will increase the denominator $R$ in equation 6 , severely affecting $Q$ unless compensatory mechanisms, such as recruitment of additional collateral venous outflow pathways become active (Figure 3A-C). ${ }^{51}$ Impaired venous outflow will result in engorgement of upstream cortical veins, reduce CPP, direct- ly affect Starling forces at the capillary bed and perturb reabsorption of fluids and solutes $^{5,35,52,53}$ (Figure 3D-F).

\section{Interstitial fluid}

The brain ECS accounts for about 15$20 \%$ of the total brain volume. ${ }^{54}$ In an average $1400 \mathrm{~mL}$ of brain weight, $210-280 \mathrm{~mL}$ is made up of ECS. The geometry of ECS is characterized by tortuous spaces surrounding neural and glial cells. ECS contains ISF and is made up of ECM, which is attached to cell membranes. The most important constituents of ECM are glycosaminoglycans, proteoglycans (e.g. hyaluronic acid), and glycoproteins (e.g. laminins, collagen, fibronectin) creating a negatively charged environment to maintain a well-hydrated ambient for cellular communication and transport of substances. ${ }^{55}$ The cellular composition of the brain differs from region to region creating important changes to diffusion properties which would either facilitate or further impede ISF movement. ${ }^{54} \mathrm{~A}$ damaged ECS will hinder flow of ISF thereby disrupting the electrochemical environment
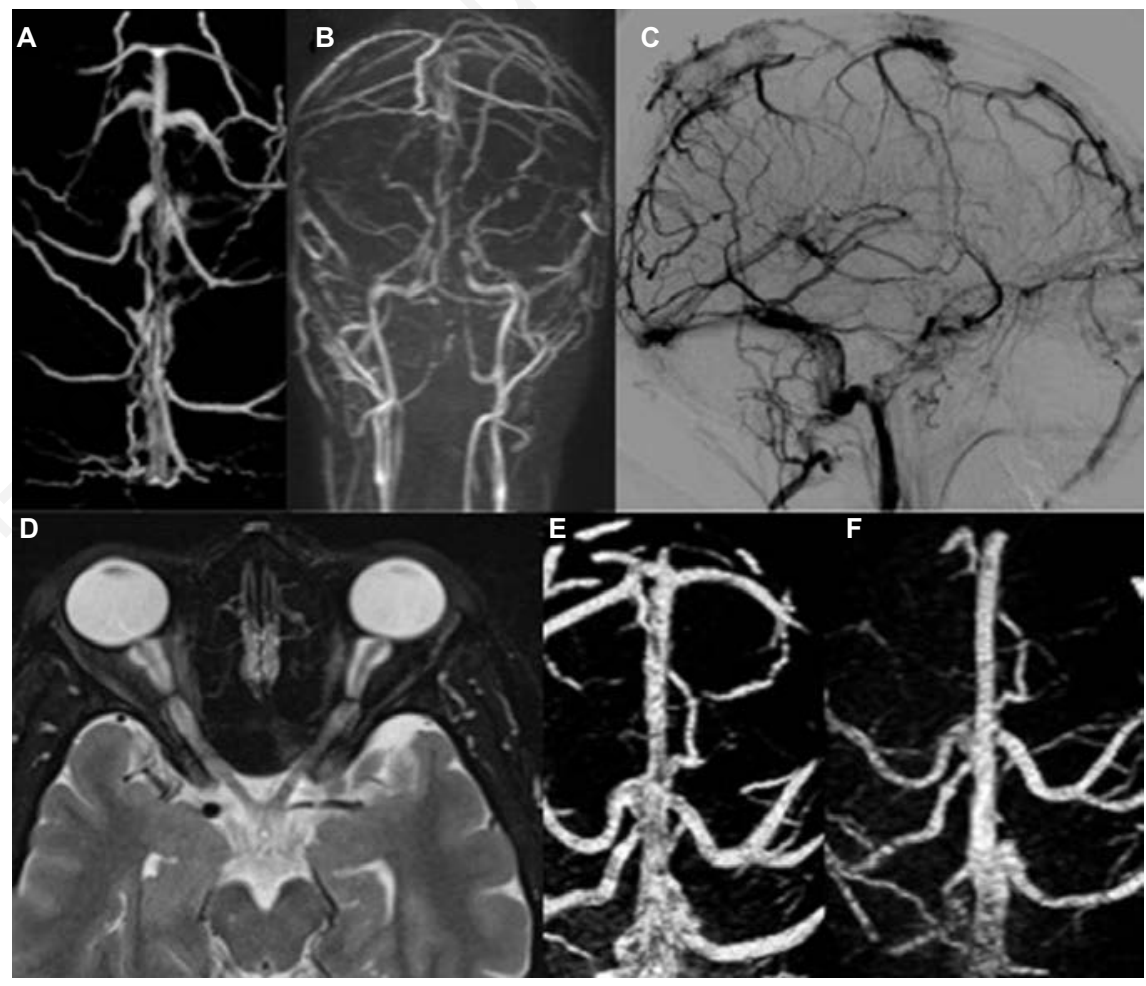

$\mathbf{F}$

Figure 3. Venous pathology. Top row: 26yr old $M$ with headaches and visual acuity loss with chronic venous thrombosis A) CE-MRV shows multiple stenosis of the SSS, irregular lumen of the BV which are puffed at the junction with the SSS; B) phase contrast coronal MRV shows multiple anastomosis of the intra and extracranial veins; $C$ ) digital subtraction angiography in the venous phase confirms massive anastomosis between the intra and extracranial veins due to thrombosed dural venous sinuses. Middle row: a $34 y r$ old F with a diagnosis of IIH D) bilateral papilledema in IIH, E) increased lumen of SSS and all BV due to transverse sinus (TS) stenosis before stent placement in the TS, and F) reduction in the lumen (and thus volume) of the SSS and BV after stenting of the TS. Bottom row: $25 y r$ old $F$ with a diagnosis of intracranial hypotension. 
water channels lined astrocytes. The CSF mixes with ISF and this fluid exits the space around veins, or venular perivascular space (VPS) (Figure 1). ${ }^{7}$ This paravascular movement of fluids and solutes is probably facilitated by arterial pulsations and respiration and represent the principal drivers of bulk flow, although recent evidence demonstrates diffusion within the ECS to be more likely. ${ }^{59-61}$ An alternative model suggests that CSF enters BP through BM within the walls of penetrating arteries whereas neurotoxic waste such as amyloid $\beta$ and $\tau$ is removed via ISF drainage along the BM, assisted by contractile activity of the SMC (aka vasomotion) in an opposite direction to arterial flow towards the deep cervical lymph nodes, also known as the intramural periarterial drainage (IPAD) ${ }^{9,62,63}$ (Figure 1). Computational modeling of IPAD suggests that such movement of ISF is faster than diffusion through BP. ${ }^{64}$

\section{Brain parenchyma}

With over 100 billion neurons, the brain is one of the most metabolically active, high-energy demanding organ in the human body. ${ }^{65}$ Oxygen and glucose are primary substrates for the production of high energy adenosine triphosphate molecules (ATP) that assure the functioning of $\mathrm{Na}^{+}-\mathrm{K}^{+}$ ATPase pumps, necessary to maintain the integrity of cell membranes and also to generate action potentials that form the basis of all neuronal activity. This activity continues relentlessly at all times including sleep. ${ }^{66}$ Since glucose is not stored in the brain, a steady supply of primary substrates is necessary for normal brain function and warrants an adequate $\mathrm{CPP}$ and $\mathrm{CBF}$ at all times. Interruption of CBF for just 10 seconds can cause neuronal dysfunction and if not restored promptly, irreversible damage can occur in a matter of a few minutes. ${ }^{67}$

BP consists of gray matter (GM) and white matter (WM) (Figure 2E). There is an outer layer of cortical GM and an inner mass of WM. Within the depths of WM, there are additional nuclei of cells, also known as subcortical gray matter nuclei and are largely represented by basal ganglia, thalami, and hippocampi. GM is a more hydrophilic environment that contains a larger quantity of fluid, is less tightly packed with cells and has a higher capillary density reflective of its elevated energy needs. ${ }^{68}$ MRI based measurements have quantified a total CBV (arterial and venous) in the GM to be about $5.5 \mathrm{~mL}$ and in the $\mathrm{WM}$ to be approximately $1.5 \mathrm{~mL}$ of every $100 \mathrm{~mL}$ of brain weight. ${ }^{69} \mathrm{WM}$, on the other hand, is made up of heavily myelinated tracts creating a highly anisotropic environment that restricts the movement of water molecules in the direction of least resistance. The mechanical and visco-elastic properties (brain compliance) of the BP vary and depend on cellular morphology, capillary distribution, the compactness of white matter axons, their orientation and ECM composition. ${ }^{70}$ These properties are different both at a macroscale (WM is stiffer than GM) and at a microscale (cortical GM is stiffer than hippocampal GM; WM in the corpus callosum is stiffer than WM in the corona radiata). ${ }^{70}$ Brain compliance is a measure that expresses the brain pressure-volume relationship. In a wellcompliant BP, changes in volume will not affect pressure until it reaches a certain threshold, whereby even small volume changes would result in very large ICP changes. ${ }^{71}$ Such characteristics are constantly changing with water content in the brain, during both neurodevelopment in early stages of life and neurodegeneration in later stages of life and will affect brain compliance. $^{72}$ In an early MRI-based study, Greitz et al. observed brain tissue pulsatile movement caused by systolic arterial expansion, noting that re-modelling of the brain enables its piston-like action that aids CSF venting to SSAS and compression of ventricles, thus promoting intra-ventricular flow. They identify cranio-spinal dynamics, as the interplay resulting from the spatial requirements of $\mathrm{BP}$ and neurofluids. Brain compliance can be influenced by age-related changes, pathological processes and also by simple manual external compression of IJVs, no doubt BP dynamics must be part of any study concerned with cranio-spinal dynamics in a holistic setting, as advocated here. $^{73}$

\section{Craniospinal inter-compartment fluid dynamics and waste clear- ance}

Main tasks of the brain fluid-mediated transport systems are: i) to supply oxygen and nutrients to sustain high-energy demands of the brain; and ii) to collect and remove metabolic waste efficiently, avoiding an accumulation of neurotoxic materials in the brain's delicate structures. The first task involves the arterial system providing fast advective transport of solutes in the blood, which is then partly delivered at the level of the microvasculature involving various forms of transport across the vessel wall. As indicated by the Einstein spacetime relation for diffusion, time is propor- tional to the square of the distance $\left(t \propto x^{2}\right)$ which means that diffusion is hopelessly slow for long distances but fast for very short distances. The thickness of brain capillary walls is of the order of $1 \mu \mathrm{m}$ and therefore in this situation, diffusion is sufficiently fast.

Transport across the vessel wall is a two-way process; in addition to the delivery of oxygen and nutrients from lumen to tissue, there is absorption into the bloodstream across the capillary/venule wall. Many diseases are likely in part due to disturbance of the two types of transport: i) fast, longrange advective transport dominated by pressure gradients; and ii) slow, shortrange, diffusion dominated transport. The integrity of the intricate morpho-functional aspects of neurofluids and BP are major determinants for the smooth delivery of vital substances for neuronal functioning and for effective transport of metabolic waste. Altered fluid dynamics will result in altered pressure, altered flow, altered wall shear stresses, altered perfusion, altered absorption, altered clearance of metabolic waste, edema, accumulation and deposition of materials in the vessel wall and the BP. Neurofluid dynamics are thus closely coupled to waste clearance mechanisms (Figure 1). A major clinical implication of such combined transport systems is their role in clearance of metabolic waste and neurotoxic substances such as amyloid $\beta$ and $\tau$ and their potential biophysical link of neurofluid dynamics to neurological diseases, such as Alzheimer's disease (AD), Parkinson's disease (PD), idiopathic intracranial hypertension (IIH), Ménière's disease (MD), multiple sclerosis (MS), migraine and other neurodegenerative diseases. ${ }^{74}$ There are however many questions to be answered with regards to brain waste clearance systems. For example, the forces responsible for the transport of CSF into the BP, exit route through the VPS and the character of such transport, advective or diffusive remain controversial and yet to be identified.

Perhaps even more challenging, is the interaction of these fluid transport systems with the venous system, especially in pathophysiological conditions. This point, to the authors' knowledge, has not yet been addressed. Here we anticipate some points that might be of interest to consider in craniospinal venous pathological conditions. First, recall that venous system: i) is a sink to CSF via arachnoid-villi drainage into the superior sagittal sinus; ii) collects ISF and solutes that is reabsorbed across vessel walls of capillary and venules; and iii) CSF/ISF flows into the deep cervical lymph nodes which empties lymph into the subcla- 
vian veins between the internal and the external jugular vein junctions. ${ }^{75}$ This powerful role of the venous system cannot be undermined and must be incorporated in the global picture of waste drain mechanisms. A thorough quantitative investigation of APS and VPS in BP is needed and seems to be lacking at present. From the point of view of the clearance capacity of the lymphatic system, the size of the VPS and its variability under pathological conditions seems most important. Transmural pressure across venular walls is the difference between pressure outside the venular wall (external pressure) and the pressure from inside the venules (internal pressure). ${ }^{76}$ In this regard, it is reasonable to suggest that in conditions of cerebral venous hypertension, venous engorgement from the inside and increased ICP from the outside would contrive to reduce the size of the VPS. Such changes will occur in cases of increased pressure in the intra or extracranial venous vessels. ${ }^{76,77}$ An obvious consequence of this would the hampering of fluid and solute transport proposed by Iliff et al. ${ }^{7}$

\section{Clinical implications}

The most complex morphological and functional structure of the brain in which the microvasculature is embedded is the neurovascular unit (NVU), made up of neurons, astrocytes, and the vascular structures. The coupling of astrocytes and neurons in the delivery of glucose and oxygen and their combustion into ATP, the molecular processes underlying the constant and prompt turnover of neurotransmitters, the processes underlying synaptic activity, volume transmission of neurotransmitters, ions and proteins, the maintenance of a healthy ECM, ISF flow, instant dynamic removal of waste products are just some of the functions of the NVU. Damage to the NVU at any level will cause irreversible damage to processes leading to the normal functioning of the neurons.

\section{Arterial pathology}

Arterial hypertension and arteriolosclerosis will weaken the walls, increase shear stress, alter the cerebral autoregulatory mechanisms, increase arterial pulsatility, affect Windkessel mechanism and consequently reduce $\mathrm{CBF}$ and $\mathrm{CPP} .{ }^{52}$ Chronic hypoperfusion will damage NVU, cause rarefaction of $\mathrm{WM}$, accelerate demyelination, damage ECM, disintegrate the BBB, $\mathrm{SMC}$, and pericytes, resulting in lacunar infarcts all of which are characteristic of small vessel disease (SVD) and leukoaraio- sis $^{78-80}$ SVD is also characterized by dilated PVS, considered a biomarker of reduced drainage of ISF and solutes and correlate directly with increased risk of vascular dementia. ${ }^{79}$ Gliosis increases tortuosity of the ECS and reduces brain compliance. ${ }^{54}$ Failure of elimination of proteins and ISF along arterial walls via IPAD can occur in a variety of genetic diseases such as cerebral autosomal dominant arteriopathy and subcortical inflammatory leukoencephalopathy, cerebral amyloid angiopathy and neurodegenerative diseases (e.g. AD). ${ }^{81}$ Arteriole walls become fragile and prone to hemorrhage, there is increased amyloidbeta deposition within the BP and arterial walls, that lead to a cascade of events and finally neuronal degeneration, a mechanism common to protein elimination failure arteriopathies and dementia. ${ }^{82}$

\section{Venous pathology}

The role of veins in neurological diseases was brought to the forefront by Zamboni, ten years ago where he found a significantly increased number of patients with multiple sclerosis with inefficient extracranial venous drainage, also termed chronic cerebrospinal venous insufficiency (CCSVI). ${ }^{83}$ Various degree of CCSVI can cause retrograde reflux of venous blood, increased intracranial venous pressure and local venous hypertension, increase CSF pulsatility in the AoS, reduce clearance of ISF, promote iron deposition, inflammation, breakdown of BBB and myelin disintegra- tion. ${ }^{74}$ Higher number of venous collaterals is present in MS patients with CCSVI as a physiological compensatory response to reduced venous output. ${ }^{84}$

There is a direct interdependency between cerebral venous flow and CSF dynamics such that intra and/or extracranial strictures will directly affect CSF flow parameters within the ventricular system in normal and pathological conditions. ${ }^{4}$ In healthy subjects MR elastography and PCMRI techniques have demonstrated that compression of IJVs in the neck directly increases CSF pulsatility in the AoS, decreases $\mathrm{CBF}$ and, increases brain tissue stiffness. ${ }^{85-87}$ The most striking evidence of such interdependency was communicated by Hans Queckenstedt more than 100 years ago (1916), modern confirmations of which are contained in the work of Alperin et al., who in the context of IIH discovered the presence of both intracranial and extracranial venous strictures that impeded normal venous drainage thereby increasing intracranial venous volume and overall altered intracranial dynamics, with raised ICP as one consequence. ${ }^{88,89}$ Extracranial IJV compression was recently proposed as a direct cause of dilated ventricles and increased ICP in a patient in a possibly new syndrome which the authors call the JEDI syndrome (jugular entrapment, dilated ventricles, intracranial hypertension). ${ }^{90}$ Intracranial venous strictures can also affect cerebral venous outflow. An example of such strictures is the idiopathic intracranial
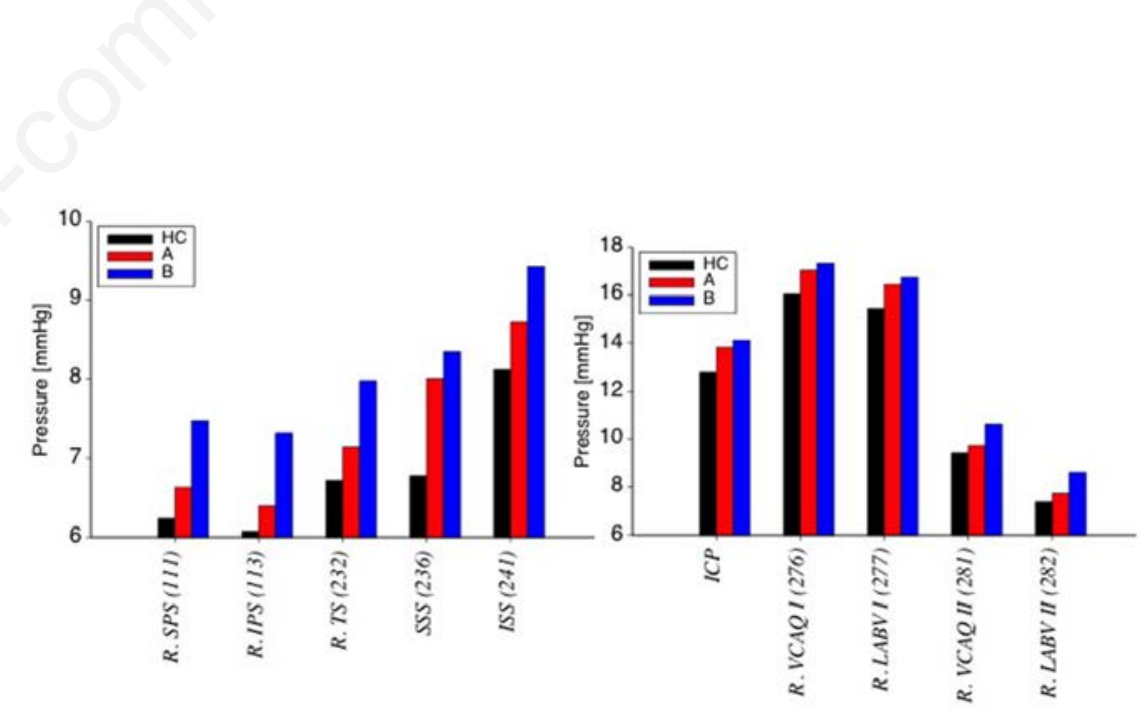

Figure 4. Impact of extracranial venous outflow strictures. Computational results from a validated global, closed-loop mathematical model show that the entire fluid dynamics of the craniospinal system is altered, including raised ICP and increased intracranial venous pressure. The figure shows computed cardiac-cycle averaged pressures for two anomalous cases A and B, compared to the healthy control (HC). The left frame shows pressures for superior petrosal sinus (SPS), inferior petrosal sinus (IPS), transverse sinus (TS), superior sagittal sinus (SSS) and inferior sagittal sinus (ISS). The right frame shows pressures in brain parenchyma (ICP), vein of the cochlear aqueduct (VCAQ) and labyrinthine vein (LABV). Reproduced from Toro et al., 2018. ${ }^{104}$ 
hypertension (IIH) characterized by a high prevalence of dural venous sinus stenosis (in particular, transverse sinus). ${ }^{91,92}$ Studies consistently report increased CSF pulsatility in the AoS and reduced CBF, both parameters that can be improved after high volume CSF removal during a lumbar puncture or venous stenting but not medical treatment alone. ${ }^{53}$ This knowledge is leading the path towards newer and efficacious treatments such as venous angioplasty and stenting. ${ }^{93}$

\section{Alterations of CSF and ISF dynamics}

In CSF circulation disorders such as normal pressure hydrocephalus (NPH), PCMRI have repeatedly shown increased CSF pulsatility in the AoS, reduced vascular compliance and decreased arterial perfusion within the BP. ${ }^{94}$ Increased brain stiffness, gliosis, and demyelination of the periventricular white matter are reflective of a more global alteration that affects transependymal flow, macromolecule transependymal transport and overall neurofluid interactions including CSF and ISF. ${ }^{10,95,96}$ The role of ISF flow has not yet gained sufficient attention in the medical world, mostly because it is hard to visualize and image the ECS and its contents. ECS is a dynamic space greatly affected by the physiologic activity of the astrocytes and neurons but also by physiologic states such as sleep. ${ }^{97}$ Stagnant ISF, impaired drainage of solutes, dilation of PVS are known to cause atrophy of the surrounding axons leading to a vicious cycle of neurodegeneration..$^{79,98}$

\section{Meningeal lymphatics pathology}

The recent discovery of meningeal lymphatics and its drainage pathway within the outer meningeal layers surrounding the dural venous sinus has been revolutionary and since then a number of neurological diseases have been shown to have altered lymphatic drainage. ${ }^{6}$ In essence, solute clearance through the CSF is achieved via drainage into meningeal lymphatics that reach deep cervical lymph nodes. Damage to these pathways, including experimental ligation of deep cervical lymph nodes can cause neurodegeneration in particular $\mathrm{AD}$, aggravate $\mathrm{PD}$ and worsen stroke outcome. ${ }^{99}$ 101

In quantitative science we either measure or we compute. Real-life measurements are difficult and even impossible since they tend to involve invasive methodology. Müller and Toro developed a comprehensive mathematical model for all major full-body fluids, with special attention paid to craniospinal fluids and the venous system in particular. ${ }^{102}$ This model has been used to study the effect of extracranial venous outflow strictures and has demonstrated increased cerebral venous pressure, increased ICP and disturbed overall cranio-spinal dynamics ${ }^{77,103}$ (Figure 4). These theoretical results have confirmed partial previous experimental results and from now on they should be regarded as fact and no longer a matter of opinion or supposition.

\section{Conclusions}

Clinicians and researchers tend to focus on single isolated fluid systems, overshadowing the broader picture of neurofluid dynamics within the brain. New observations and developments in neuroscience force us to revise our old dogmas and challenge us to adopt new concepts. By interrogating different physiologic mechanisms that may have been jeopardised in a disease at any one time, understanding the dynamic nature of the entire system and its individual compartments, is likely the only way to find answers to the several neurological diseases that still remain without a specific etiology and treatment (including several idiopathic and atypical variants of diseases). The venous system in the brain has not yet been carefully studied in a clinical setting and requires urgent attention. Veins are the sink of the entire fluid content in the brain and it would be irrational to not comprehend the extent of their involvement in neurological diseases and to revisit treatment options. Potential consequences still to be established include decreased cerebral perfusion and disturbed CNS clearance system as a result of venous strictures.

\section{References}

1. Feigin VL, Abajobir AA, H K, et al. Global, regional, and national burden of neurological disorders during 19902015: a systematic analysis for the Global Burden of Disease Study 2015. Lancet Neurol 2017;16:877-97.

2. Kellie G. An account of the appearances observed in the dissection of two of three individuals presumed to have perished in the storm of the $3 \mathrm{~d}$, and whose bodies were discovered in the vicinity of Leith on the morning of the 4th, November 1821: with some reflections on the pathology of the brain. Royal College of Surgeons of England; 1824. Available from: https://archive.org/details/b22384315

3. Mokri B. The Monro-Kellie hypothesis: applications in CSF volume deple- tion. Neurology 2001;56:1746-8.

4. Beggs CB. Cerebral venous outflow and cerebrospinal fluid dynamics. Veins and Lymphatics 2014;3:1-8.

5. Wilson MH. Monro-Kellie 2.0: The dynamic vascular and venous pathophysiological components of intracranial pressure. J Cereb Blood Flow Metab 2016;36:1338-50.

6. Louveau A, Smirnov I, Keyes TJ, et al. Structural and functional features of central nervous system lymphatic vessels. Nature 2015;523:337-41.

7. Iliff JJ, Wang M, Liao Y, et al. A paravascular pathway facilitates CSF flow through the brain parenchyma and the clearance of interstitial solutes, including amyloid $\beta$. Sci Transl Med 2012;4:147ra111-147ra111.

8. Benveniste H, Lee H, Volkow ND. The Glymphatic Pathway: Waste Removal from the CNS via Cerebrospinal Fluid Transport. Neurosci 2016;23:454-65.

9. Carare RO, Bernardes-Silva M, Newman TA, et al. Solutes, but not cells, drain from the brain parenchyma along basement membranes of capillaries and arteries: significance for cerebral amyloid angiopathy and neuroimmunology. Neuropathol Appl Neurobiol 2008;34:131-44.

10. Linninger AA, Tangen K, Hsu C-Y, Frim D. Cerebrospinal Fluid Mechanics and Its Coupling to Cerebrovascular Dynamics. Annu Rev Fluid Mech 2016;48:219-57.

11. Zhang ET, Inman CBE, Weller RO. Interrelationships of the pia mater and the perivascular (Virchow-Robin) spaces in the human cerebrum. J Anat 1990;170:111-23.

12. Weller RO. Microscopic morphology and histology of the human meninges. Morphologie 2005;89:22-34.

13. Marín-Padilla M. The human brain intracerebral microvascular system: development and structure. Front Neuroanat 2012;6:38. eCollection 2012.

14. Abbott NJ, Pizzo ME, Preston JE, et al. The role of brain barriers in fluid movement in the CNS: is there a "glymphatic" system? Acta Neuropathol 2018;135:1-21.

15. Pizzo ME, Wolak DJ, Kumar NN, et al. Intrathecal antibody distribution in the rat brain: surface diffusion, perivascular transport and osmotic enhancement of delivery. J Physiol 2017;596:44575.

16. Hill RA, Tong L, Yuan $\mathrm{P}$, et al. Regional Blood Flow in the Normal and Ischemic Brain Is Controlled by Arteriolar Smooth Muscle Cell 
Contractility and Not by Capillary Pericytes. Neuron 2015;87:95-110.

17. Hamel E. Perivascular nerves and the regulation of cerebrovascular tone. J Appl Physiol 2006;100:1059-64.

18. Zanatta P, Toffolo GM, Sartori E, et al. The human brain pacemaker: Synchronized infra-slow neurovascular coupling in patients undergoing nonpulsatile cardiopulmonary bypass. NeuroImage 2013;72:10-9.

19. Hall CN, Reynell C, Gesslein B, et al. Capillary pericytes regulate cerebral blood flow in health and disease. Nature 2014;508:1-16.

20. Fantini S, Sassaroli A, Tgavalekos KT, Kornbluth J. Cerebral blood flow and autoregulation: current measurement techniques and prospects for noninvasive optical methods. Neurophoton 2016;3:031411-32.

21. Hawkins BT, Davis TP. The bloodbrain barrier/neurovascular unit in health and disease. Pharmacol Rev 2005;57:173-85.

22. Furuse M. Molecular basis of the core structure of tight junctions. Cold Spring Harb Perspect Biol 2010;2:a002907-a002907.

23. Wong AD, Mao Y, Levy AF, et al. The blood-brain barrier: an engineering perspective. Front Neuroeng 2013;6:7.

24. Mantovani G, Menegatti M, Scerrati A, et al. Controversies and Misconceptions Related to Cerebrospinal Fluid Circulation: A Review of the Literature from the Historical Pioneers' Theories to Current Models. BioMed Res Int 2018;2018:1-7.

25. Faraci FM, Mayhan WG, Heistad DD. Effect of vasopressin on production of cerebrospinal fluid: possible role of vasopressin (V1)-receptors. Am J Physiol 1990;258:R94-R98.

26. Mortamet B, Zeng D, Gerig G, et al. Effects of healthy aging measured by intracranial compartment volumes using a designed MR brain database. Med Image Comput Comput Assist Interv 2005;8:383-91.

27. Czosnyka M, Czosnyka Z, Momjian S, Pickard JD. Cerebrospinal fluid dynamics. Physiol Measure 2004;25:R51-R76.

28. Laterra J, Keep R, Betz LA, Goldstein GW. Blood - Cerebrospinal Fluid Barrier. In: Basic Neurochemistry: Molecular, Cellular and Medical Aspects. 6th Edition. Sixth. LippincottRaven; 1999.

29. Spector R, Keep RF, Snodgrass SR, et al. A balanced view of choroid plexus structure and function: Focus on adult humans. Exper Neurol 2015;267:7886.

30. Brinker T, Stopa E, Morrison J, Klinge P. A new look at cerebrospinal fluid circulation. Fluids Barriers CNS 2014;11:10.

31. Weller RO, Kida S, Zhang ET. Pathways of fluid drainage from the brain-morphological aspects and immunological significance in rat and man. Brain Pathol 1992;2:277-84.

32. de Leon MJ, Li Y, Okamura N, et al. Cerebrospinal Fluid Clearance in Alzheimer Disease Measured with Dynamic PET. J Nuclear Med 2017;58:1471-6.

33. Aspelund A, Antila S, Proulx ST, et al. A dural lymphatic vascular system that drains brain interstitial fluid and macromolecules. J Exp Med 2015;212:991-9.

34. Absinta M, Ha S-K, Nair G, et al. Human and nonhuman primate meninges harbor lymphatic vessels that can be visualized noninvasively by MRI. Elife 2017;6:780.

35. Orešković D, Radoš M, Klarica M. New Concepts of Cerebrospinal Fluid Physiology and Development of Hydrocephalus. Pediatr Neurosurg 2016; (0): 1-9.

36. Greitz D, Hannerz J. A proposed model of cerebrospinal fluid circulation: observations with radionuclide cisternography. AJNR Am J Neuroradiol 1996;17:431-8.

37. Casaca-Carreira J, Temel Y, Hescham S-A, Jahanshahi A. Transependymal Cerebrospinal Fluid Flow: Opportunity for Drug Delivery? Mol Neurobiol 2018;55:1-9.

38. Han H, Li K, Yan J, et al. An in vivo study with an MRI tracer method reveals the biophysical properties of interstitial fluid in the rat brain. Sci China Life Sci 2012;55:782-7.

39. Dreha-Kulaczewski S, Joseph AA, Merboldt K-D, et al. Identification of the Upward Movement of Human CSF In Vivo and its Relation to the Brain Venous System. J Neurosci 2017;37:2395-402.

40. Stoquart-El Sankari S, Lehmann P, Gondry-Jouet C, et al. Phase-Contrast MR Imaging Support for the Diagnosis of Aqueductal Stenosis. Am J Neuroradiol 2008;30:209-14.

41. Sakhare AR, Barisano G, Pa J. Assessing test-retest reliability of phase contrast MRI for measuring cerebrospinal fluid and cerebral blood flow dynamics. Magn Reson Med 2019;82:658-70.

42. Andeweg J. The anatomy of collateral venous flow from the brain and its value in aetiological interpretation of intracranial pathology. Neuroradiology 1996;38:621-8.

43. Ayanzen RH, Bird CR, Keller PJ, et al. Cerebral MR venography: normal anatomy and potential diagnostic pitfalls. AJNR Am J Neuroradiol 2000;21:74-8.

44. Kiliç T, Akakin A. Anatomy of cerebral veins and sinuses. Front Neurol Neurosci 2008;23:4-15.

45. Nakai K, Imai H, Kamei I, et al. Microangioarchitecture of rat parietal cortex with special reference to vascular "sphincters." Scanning electron microscopic and dark field microscopic study. Stroke 1981;12:653-9.

46. Vignes J-R, Dagain A, Guérin J, Liguoro D. A hypothesis of cerebral venous system regulation based on a study of the junction between the cortical bridging veins and the superior sagittal sinus. Laboratory investigation. J Neurosurg 2007;107:1205-10.

47. Batson OV. The function of the vertebral veins and their role in the spread of metastases. Ann Surg 1940;112:13849.

48. Doepp F, Schreiber SJ, Münster von T, et al. How does the blood leave the brain? A systematic ultrasound analysis of cerebral venous drainage patterns. Neuroradiol 2004;46:565-70.

49. Kitano M, Oldendorf WH, Cassen B. The elasticity of the cranial blood pool. J Nucl Med 1964;5:613-25.

50. Nakakita K, Imai H, Kamei I, et al. Innervation of the cerebral veins as compared with the cerebral arteries: a histochemical and electron microscopic study. J Cereb Blood Flow Metab 1983;3:127-32.

51. Chou C-H, Doong M-L, Fuh J-L, et al. Queckenstedt's Test Affects More than Jugular Venous Congestion in Rat. Mukhopadhyay P, ed. PLoS One 2013;8:e59409-6.

52. Bateman GA. Arterial inflow and venous outflow in idiopathic intracranial hypertension associated with venous outflow stenoses. J Clin Neurosci 2008;15:402-8.

53. Agarwal N, Contarino C, Limbucci N, et al. Intracranial Fluid Dynamics Changes in Idiopathic Intracranial Hypertension: Pre and Post Therapy. CNR 2018;15:164-72.

54. Syková E, Nicholson C. Diffusion in brain extracellular space. Physiol Rev 2008;88:1277-340.

55. Crocker SJ, Pagenstecher A, Campbell IL. The TIMPs tango with MMPs and more in the central nervous system. J 
Neurosci Res 2004;75:1-11.

56. Agnati LF, Zoli M, Stromberg I, Fuxe $\mathrm{K}$. Intercellular communication in the brain: wiring versus volume transmission. NSC 1995;69:711-26.

57. Abbott NJ. Evidence for bulk flow of brain interstitial fluid: significance for physiology and pathology. Neurochem Int 2004;45:545-52.

58. Cserr HF. Role of secretion and bulk flow of brain interstitial fluid in brain volume regulation. Ann New York Acad Sci 1988;529:9-20.

59. Iliff JJ, Wang M, Zeppenfeld DM, et al. Cerebral Arterial Pulsation Drives Paravascular CSF-Interstitial Fluid Exchange in the Murine Brain. J Neurosci 2013;33:18190-9.

60. Kiviniemi V, Wang X, Korhonen V, et al. Ultra-fast magnetic resonance encephalography of physiological brain activity - Glymphatic pulsation mechanisms? J Cereb Blood Flow Metab 2016;36:1033-45.

61. Holter KE, Kehlet B, Devor A, et al. Interstitial solute transport in $3 \mathrm{D}$ reconstructed neuropil occurs by diffusion rather than bulk flow. Proc Natl Acad Sci U S A 2017;114:9894-9.

62. Diem AK, MacGregor Sharp M, Gatherer M, et al. Arterial Pulsations cannot Drive Intramural Periarterial Drainage: Significance for $A \beta$ Drainage. Front Neurosci 2017;11:353-9.

63. Aldea R, Weller RO, Wilcock DM, et al. Cerebrovascular Smooth Muscle Cells as the Drivers of Intramural Periarterial Drainage of the Brain. Front Aging Neurosci 2019;11:253-17.

64. Diem AK, Tan M, Bressloff NW, et al. A Simulation Model of Periarterial Clearance of Amyloid- $\beta$ from the Brain. Front Aging Neurosci 2016;8:545-11.

65. Navarrete A, van Schaik CP, Isler K. Energetics and the evolution of human brain size. Nature 2011;480:91-3.

66. Raichle ME. The restless brain: how intrinsic activity organizes brain function. Philos Trans Roy Soc B Biol Sci 2015;370:20140172-20140172.

67. Hossmann K-A. Pathophysiology and therapy of experimental stroke. Cell Mol Neurobiol 2006;26:1055-81.

68. Zhu X-H, Qiao H, Du F, et al. Quantitative imaging of energy expenditure in human brain. NeuroImage 2012;60:2107-17.

69. Lu H, Law M, Johnson G, et al. Novel approach to the measurement of absolute cerebral blood volume using vascular-space-occupancy magnetic resonance imaging. Magn Reson Med
2005;54:1403-11.

70. Lee SJ, King MA, Sun J, et al. Measurement of viscoelastic properties in multiple anatomical regions of acute rat brain tissue slices. J Mechan Behav Biomed Mater 2014;29:213-24.

71. Czosnyka M, Citerio G. Brain compliance: the old story with a new 'etcetera'. Intensive Care Med 2012;38:925-7.

72. Huang H, Zhang J, Wakana S, et al. White and gray matter development in human fetal, newborn and pediatric brains. NeuroImage 2006;33:27-38.

73. Greitz D, Wirestam R, Franck A, et al. Pulsatile brain movement and associated hydrodynamics studied by magnetic resonance phase imaging. The MonroKellie doctrine revisited. Neuroradiol 1992;34:370-80.

74. Haacke EM, Beggs CB, Habib C. The role of venous abnormalities in neurological disease. Rev Recent Clin Trials 2012;7:100-16.

75. Aspelund A, Robciuc MR, Karaman S, et al. Lymphatic system in cardiovascular medicine. Circ Res 2016;118:515-30.

76. Zamboni P. Chapter 29 - The Contribution of Extra Cranial Venous Drainage to Neuro-Inflammation in Multiple Sclerosis. In: Minagar A, ed. Neuroinflammation (Second Edition). Neuroinflammation (Second Edition). Academic Press; 2018, pp 579-599.

77. Toro EF, Müller LO, Cristini M, et al. Impact of jugular vein valve function on cerebral venous haemodynamics. CNR 2015;12:384-97.

78. Cannistraro RJ, Badi M, Eidelman BH, et al. CNS small vessel disease: A clinical review. Neurology 2019;92:114656.

79. Brown R, Benveniste H, Black SE, et al. Understanding the role of the perivascular space in cerebral small vessel disease. Cardiovasc Res 2018;114:1462-73.

80. Beggs CB, Magnano C, Shepherd SJ, et al. Dirty-Appearing White Matter in the Brain is Associated with Altered Cerebrospinal Fluid Pulsatility and Hypertension in Individuals without Neurologic Disease. J Neuroimaging 2015;26:136-43.

81. Carare RO, Hawkes CA, Jeffrey M, et al. Review: Cerebral amyloid angiopathy, prion angiopathy, CADASIL and the spectrum of protein elimination failure angiopathies (PEFA) in neurodegenerative disease with a focus on therapy. Neuropathol Appl Neurobiol 2013;39:593-611.

82. Carare RO. Editorial: Clearance
Pathways for Amyloid- $\beta$. Significance for Alzheimer's Disease and Its Therapy. Front Aging Neurosci 2017;9:396-2.

83. Zamboni P, Galeotti R, Menegatti E, et al. Chronic cerebrospinal venous insufficiency in patients with multiple sclerosis. J Neurol Neurosur Psychiatr 2008;80:392-9.

84. Haacke EM, Sethi SK, Jiang J, et al. The role of magnetic resonance imaging in assessing venous vascular abnormalities in the head and neck: a demonstration of cerebrospinal venous insufficiency in a subset of multiple sclerosis patients. Veins and Lymphatics 2015;4:1-9.

85. Hatt A, Cheng S, Tan K, et al. MR Elastography Can Be Used to Measure Brain Stiffness Changes as a Result of Altered Cranial Venous Drainage During Jugular Compression. AJNR Am J Neuroradiol 2015;36:1971-7.

86. Bhadelia RA, Bogdan AR, Wolpert SM. Cerebrospinal fluid flow waveforms: effect of altered cranial venous outflow. A phase-contrast MR flow imaging study. Neuroradiol 1998;40:283-92.

87. Beggs CB, Magnano C, Shepherd SJ, et al. Aqueductal cerebrospinal fluid pulsatility in healthy individuals is affected by impaired cerebral venous outflow. J Magn Reson Imaging 2013;40:1215-22.

88. Pearce JMS. The Craniospinal Venous System. Eur Neurol 2006;56:136-8.

89. Alperin N, Lee SH, Mazda M, et al. Evidence for the importance of extracranial venous flow in patients with idiopathic intracranial hypertension (IIH). Acta Neurochir Suppl 2005;95:129-32.

90. De Bonis P, Menegatti E, Cavallo MA, et al. JEDI (jugular entrapment, dilated ventricles, intracranial hypertension) syndrome: a new clinical entity? A case report. Acta Neurochir 2019;161:136770 .

91. Farb RI, Vanek I, Scott JN, et al. Idiopathic intracranial hypertension: The prevalence and morphology of sinovenous stenosis. Neurology 2003;60:1418-24.

92. Morris PP, Black DF, Port J, Campeau N. Transverse Sinus Stenosis Is the Most Sensitive MR Imaging Correlate of Idiopathic Intracranial Hypertension. AJNR Am J Neuroradiol 2017;38:471-7.

93. Dinkin MJ, Patsalides A. Venous Sinus Stenting in Idiopathic Intracranial Hypertension. J Neuro-Ophthalmol 2017;37:113-21. 
94. Bateman GA. Vascular compliance in normal pressure hydrocephalus. AJNR Am J Neuroradiol 2000;21:1574-85.

95. Fattahi N, Arani A, Perry A, et al. MR Elastography Demonstrates Increased Brain Stiffness in Normal Pressure Hydrocephalus. AJNR Am J Neuroradiol 2016; 37: 462-7.

96. Krishnamurthy S, Li J, Shen Y, et al. Normal macromolecular clearance out of the ventricles is delayed in hydrocephalus. Brain Res 2018;1678:33755.

97. Jessen NA, Munk ASF, Lundgaard I, Nedergaard M. The Glymphatic System: A Beginner's Guide. Neurochem Res 2015;40:2583-99.

98. Mestre H, Kostrikov S, Mehta RI,
Nedergaard M. Perivascular spaces, glymphatic dysfunction, and small vessel disease. Clin Sci 2017;131:225774.

99. Da Mesquita S, Louveau A, Vaccari A, et al. Functional aspects of meningeal lymphatics in ageing and Alzheimer's disease. Nature 2018;560:185-91.

100.Zou W, Pu T, Feng W, et al. Blocking meningeal lymphatic drainage aggravates Parkinson's disease-like pathology in mice overexpressing mutated $\alpha$ synuclein. Transl Neurodeg 2019:1-17.

101. Yanev P, Poinsatte K, Hominick D, et al. Impaired meningeal lymphatic vessel development worsens stroke outcome. J Cereb Blood Flow Metab 2019:271678X18822921.
102. Müller LO, Toro EF. Enhanced global mathematical model for studying cerebral venous blood flow. J Biomechan 2014;47:3361-72.

103. Müller LO, Toro EF, Haacke EM, Utriainen D. Impact of CCSVI on cerebral haemodynamics: a mathematical study using MRI angiographic and flow data. Phlebology 2015;31:305-24.

104. Toro EF, Borgioli F, Zhang Q, Contarino C, Müller LO, Bruno A. Inner-ear circulation in humans is disrupted by extracranial venous outflow strictures: Implications for Ménière's disease. Veins and Lymphatics 2018;7:1-12. 